\title{
Obesity, Cardiovascular Disease, and Influenza: How Are They Connected?
}

\author{
Lucia Gutiérrez-Spillari $^{1}$ • Geovani Palma M. ${ }^{2}$. Jorge Aceituno-Melgar ${ }^{3}$ \\ Published online: 6 July 2020 \\ (C) Springer Nature Switzerland AG 2020
}

\begin{abstract}
Purpose of Review To better understand the impact of obesity and cardiovascular diseases on influenza A infection. Recent Findings This infection could have detrimental outcomes in obese patients with cardiovascular diseases, such as an increased risk, length of hospitalization, disease severity, morbidity, and mortality. Nevertheless, there also might be some cardioprotective benefits associated with influenza vaccination, such as a reduced mortality, hospitalization, and acute coronary syndromes, in patients with coronary heart disease and/or heart failure.

Summary Obesity negatively impacts immune function and host defense. Recent studies report obesity to be an independent risk factor for increased morbidity and mortality following infection. Obese patients might need special considerations in the treatment; however, there is not enough evidence to fully comprehend the mechanisms behind the reduced immunocompetence when influenza A infection occurs. Future studies should focus on special consideration treatments when the patients have not been vaccinated and have cardiovascular diseases.
\end{abstract}

Keywords Obesity $\cdot$ Influenza $\cdot$ Cardiovascular disease $\cdot$ Cardioprotective effects of vaccination $\cdot$ Influenza vaccine

\section{Introduction}

This review focuses on how obesity and cardiovascular disease impact influenza response. Retrospective studies demonstrate that during the $2009 \mathrm{H} 1 \mathrm{~N} 1$ pandemic, obesity was identified as a risk factor for hospitalization, mechanical ventilation, and mortality upon infection. These data must be highlighted since it is projected that nearly $50 \%$ of the worldwide population is going to be obese by 2050 . Several case studies have identified possible effects of obesity on viral replication in the deep lung, progression to viral pneumonia, and prolonged viral shedding [1]. Therefore, management of

This article is part of the Topical Collection on Metabolism in Tropical Medicine

Lucia Gutiérrez-Spillari

Lgutierrez@ufm.edu

1 School of Clinical Nutrition, Universidad Francisco Marroquín, 6 Calle final, zona 10, Guatemala City, Guatemala

2 Endocrinology Department, Instituto Nacional de Ciencias Médicas y Nutrición Salvador Zubirán, Ciudad de México, Mexico

3 Cardiology Department, Instituto Nacional de Cardiología Ignacio Chávez, Ciudad de México, Mexico the influenza infection in this at-risk population has to have special consideration given that they may not respond optimally to vaccination [2].

\section{Obesity and Its Impact on Influenza Infection}

Excessive fat accumulation that results in obesity impairs health for adults [3]. Its low-grade chronic inflammatoryinduced state negatively impacts immune function and host defense [2], as shown during the 2009 influenza A virus H1N1 pandemic, where obesity resulted to be an independent risk factor for severe disease, hospitalization, mechanical ventilation, and mortality upon infection [1].

It is well known that influenza A virus infection is characterized by fever, myalgia, rhinorrhea, sore throat, and sneezing. These symptoms peak $3-5$ days post-infection, with viral shedding peaking at days $2-3$. Usually, it is limited to the upper respiratory tract; however, in severe cases, the lower respiratory tract, including the lungs, can be affected and often requires hospitalization. This progression is more common in obese patients, leading to diminish infection resolution when compared with non-obese patients [1]. Obesity also plays a role in the outcome of critical complications from influenza 
$\mathrm{A} / \mathrm{pdmH} 1 \mathrm{~N} 1$ infection and is associated with longer mechanical ventilation for severe acute respiratory distress syndrome and shock [4].

A higher body mass index (BMI) and metabolic syndrome in patients with influenza have shown an increased risk and length of hospitalization [4-6], increased disease severity, morbidity, and mortality during lower respiratory tract infections. This might be explained in part by increased lung permeability during infection, found in mice studies. Obese mice have an increased protein leakage from the lung into the bronchoalveolar lavage fluid when compared to lean mice. Additionally, lung edema and oxidative stress are also increased, which emphasizes the multiple etiologies of increased lung pathology in the obese host and the impairment in wound repair $[1,4]$.

The obesogenic state can also affect the influenza A virus evolution. It is well known that obese individuals are malnourished besides their excess in fat; they might also present nutrient deficiencies, such as vitamins [7], minerals, and trace elements [8]. There are a variety of mechanisms by which nutritional imbalances could alter within-host viral evolution [8]. Studies have shown that such imbalances prolong infections, delay clearance, and increase shedding (42\% longer than non-obese) [9], all of which potentially increase viral transmission [1].

In addition to the decreased immunocompetence mechanisms, other potential factors might contribute to its increased susceptibility to infection in the hospital setting. Some examples, which are underlying diseases that affect mobility, can also increase risk for skin problems, prolonged visits at hospitals and nosocomial infections, alteration in the pharmacokinetics of some drugs, and an increased susceptibility to postsurgery infections [2]. Thus, it is a complex problem that needs further evidence to develop better treatments for this increasing population.

\section{Importance of Influenza Vaccination}

Obesity causes a chronic state of inflammation in a generalized and constant way with negative effects on immunity. Obese people have delayed immune responses to influenza virus infection and experience slower recovery from the disease. In addition, the efficacy of the treatment and the vaccine is reduced in this population causing an alteration of the viral life cycle and, coupled with an already weakened and delayed immune response, leading to a more serious condition. Poor initial and adaptive responses to infection and vaccination create an impaired ability to respond appropriately to infection. The efficacy of the vaccine may decrease in obese humans; however, more studies are needed to better understand how the obese state affects infection control [1].
Previous studies suggest that the severity of influenza virus infection is multifactorial and may be related to lung spread and repair, the formation of extracellular concentrates of neutrophils at the lung level; however, this mechanism in individuals is unknown [10].

The efficacy of the vaccine in human groups has shown that initial seroconversion rates are high in the obese population, but that over time there is a greater decrease in efficacy than that observed in non-obese populations [11]. Influenza vaccine as a method of prevention is formulated each year, typically containing both influenza A and B. A study conducted in 2013-2015 aimed at evaluating whether obesity was associated with an increased risk of influenza for influenza and influenza-like illness among vaccinated obese and nonobese adults, finding that, among the obese, $9.8 \%$ had confirmed influenza or influenza-like illness compared with $5.1 \%$ of healthy weight participants. Compared with the vaccinated healthy weight, obese participants had twice the risk of developing influenza or influenza-like illness (relative risk $=2.01$, 95\% CI 1.12, 3.60, $p=0.020$ ); therefore, in this risk group, in the same way, vaccination is very important [12]. Although it appears that in high-risk groups, such as the obese and overweight population, vaccination may not provide optimal protection, and because of the growing trend of obesity worldwide, the efficacy of the vaccine should be improved [10].

Among cardiovascular disease patients, there is compelling evidence that shows lower risk of major adverse cardiovascular events, reduced hospitalization, and mortality [13-15], being the greatest treatment effect was seen among the highestrisk patients with more active coronary disease [15].

The recent recommendation advocates the priority of vaccination against influenza in obese patients; a vaccination program should be fully evaluated in obese adults. High-dose vaccines designed to vaccinate people over 65 can also be used in the obese population $[9,10]$.

\section{Cardiovascular Risk and Influenza Infection}

In the twentieth century during influenza epidemics, there has been an excess of mortality from cardiovascular disease [16]. A recent study that included 364 hospitalizations for acute myocardial infarction demonstrated an increased risk of acute myocardial infarction within one week after influenza virus infection to a risk that was six times higher than the risk during the year before or after the onset of infection [17]. Cardiovascular complications associated with influenza infection include myocarditis, pericardial effusion, myopericarditis, right and left ventricle dysfunction, myocardial infarction, heart failure, stroke, and circulatory failure due to septic shock [13, 18-20]. The risk of myocardial infarction after mild respiratory infection returns to baseline within approximately 5 weeks, but in the case of 
pneumonia complicated by sepsis the risk persists up to 10 years after the infection [16] [21].

Infectious agents (including influenza virus) have been implicated in the etiology of atherosclerosis [22]. There have been described several mechanisms by which influenza increases the risk of cardiovascular events; they may be related to pro-inflammatory mediators, sympathetic stimulation, and the activation of the coagulation cascade [19].

\section{Myocardial Infarction}

According to the fourth universal definition of myocardial infarction, there are 5 types of myocardial infarction based on clinical, electrocardiographic, and laboratory evaluation [23]. Influenza infection can trigger type 1 and type 2 myocardial infarctions [16].

Type 1 myocardial infarction is defined as myocardial ischemia caused by atherothrombotic coronary artery disease, and it is usually precipitated by atherosclerotic plaque disruption that can be rupture or erosion [23]. It is important to remember that atherosclerotic plaques also contain inflammatory cells, and pro-inflammatory cytokines, such as interleukins 1,6 , and 8 , and tumor necrosis factor $\alpha$ are generated as a response of infection. These inflammatory cytokines can activate inflammatory cells in atherosclerotic plaques [16] [24].

Acute influenza infection is associated to a procoagulant state that increases the risk of coronary thrombosis at sites of plaque disruption [16] [25, 26]. Infection with influenza virus is associated with expression of genes that have been linked to platelet activation: $\mathrm{H} 1 \mathrm{~N} 1$ exposure increases platelet gene expression signature, which is associated with myocardial infarction [25].

Type 2 myocardial infarction might be considered with a rise and/or fall of cardiac troponin values and evidence of an imbalance between myocardial oxygen supply and demand unrelated to coronary thrombosis. [23]. Influenza infection produces a systemic inflammatory response with a resulting increase in heart rate and shortens the filling time during diastole compromising in that way, the coronary blood supply. If septic shock occurs, it may have a substantial adverse effect on coronary perfusion. In older patients with chronic coronary plaques, systemic inflammation causes cardiac metabolic mismatch increasing the risk of myocardial infarction [16, 18].

\section{Heart Failure}

Influenza infection is also associated with increased mortality in patients with heart failure [27] and is vulnerable to influenza-associated complications [28] [29]. This type of patients has limited cardiac and respiratory reserves and may not tolerate the metabolic demand and hypoxemia, exacerbating underlying cardiac disease probably due to an increased sympathetic nervous system activity, hypoxemia, and renal dysfunction that can lead to volume overload [28].

In a healthy heart, severe acute influenza infection produces pro-inflammatory cytokine level elevations that can cause acute myocarditis [28] [18], characterized by a broad spectrum of symptoms that go from asymptomatic courses to signs of myocardial infarction to devastating illness with cardiogenic shock [30] [31]. Myocarditis often results from common viral infections and post-viral immune-mediated responses [30]. In acute myocarditis, there is a high incidence of wall motion abnormalities. During influenza epidemics, $15 \%$ of the patients admitted to a military hospital with influenza infection had wall motion abnormalities on echocardiogram [18]. This becomes important, due to the fact that the myocardium inflammatory disease is regarded as a precursor of dilated cardiomyopathy [30] [32]. The electrocardiographic findings in patients with myocarditis range from nonspecific T-wave and ST segment changes to ST segment elevation resembling an acute myocardial infarction; supraventricular and ventricular arrhythmias can also be present. Electrocardiographic findings that are related to poor clinical outcome include a QTc prolongation at $440 \mathrm{~ms}$, an abnormal QRS axis, and ventricular ectopic beats [30].

A recent study developed to evaluate the incidence and hemodynamic consequences of right ventricular and left ventricular dysfunction in patients with $\mathrm{H} 1 \mathrm{~N} 1$ infection demonstrated that on admission $72 \%$ had abnormal ventricular function (46\% had isolated left ventricular abnormalities and 39\% had isolated right ventricular dysfunction) and $14 \%$ had biventricular dysfunction. On the follow-up, right ventricular function tended to worsen during hospitalization, but left ventricular function tended to normalize. However, patients with ventricular dysfunction needed more aggressive therapy and of rescue ventilatory strategies, such as inhaled nitric oxide, prone positioning, and extracorporeal membrane oxygenation [18].

\section{Stroke}

During influenza epidemics, hospitalizations for cerebrovascular diseases increase [33]. An increase in incidence of ischemic stroke within 2 weeks after influenza infection has been suggested [34, 35]. Protein C pathway and endogenous fibrinolysis are mechanisms associated with cerebrovascular ischemia and influenza infection [19]. Influenza infection develops a prothrombotic state by increasing tissue factor expression and decreases fibrinolytic capacity by increased plasminogen-activator inhibitor-1 (PAI-1) expression. This results in an unbalance between coagulation and anticoagulant pathways [36].

As in myocardial infarction, the relationship between systemic inflammation and stroke pathophysiology has shown 
that stroke often occurs in a pre-existing state of inflammation due to atherosclerosis, obesity, or infection [37].

\section{Possible Cardioprotective Effects of Influenza Vaccination}

The Sixth Joint Task Force of the European Society of Cardiology and Other Societies on Cardiovascular Disease Prevention in Clinical Practice recommend that annual influenza vaccination can be considered in patients with established cardiovascular disease (class IIb, level C) [38], based on the fact that the risk of a cardiovascular event (myocardial infarction or stroke) is more than four times higher after a respiratory tract infection, with the highest risk in the first 3 days after infection [39]. There have been developed several studies that demonstrate that influenza vaccination reduces mortality, hospitalization, and acute coronary syndromes in patients with coronary heart disease and/or heart failure [13].

The mechanisms by which acute inflammation affect the risk of vascular events include the following: endothelial dysfunction, procoagulant state, and inflammatory changes in atherosclerotic plaques $[39,40]$. It is also known that persistent systemic inflammatory activity is a risk of factor for cardiovascular disease, and higher interleukin-6 blood levels increase cardiovascular mortality at one year after pneumonia infection [41]. This systemic inflammatory response can be reduced by vaccination [13]. When estimating the cost and benefit of interventions to prevent pneumonia, the association of pneumonia with cardiovascular disease risk should also be considered [40].

Patients with chronic heart failure are vulnerable to influenza-related complications (including secondary infections, such as pneumonia and acute heart failure exacerbations). Recently, the PARADIGM-HF Trial assessed associations between receiving influenza vaccine and cardiovascular death or heart failure hospitalizations, all-cause hospitalizations, and cardiopulmonary or influenza-related hospitalizations, concluding that vaccination was associated with reduced risk for death [42].

There have been described two possible mechanisms by which influenza vaccination may reduce cardiovascular events: unspecific and specific effects [13]. The unspecific mechanism is based on the fact that influenza infection causes a systemic inflammatory response, endothelial dysfunction, and a procoagulant state. These factors have negative effects on patients with previous cardiovascular diseases, such as ischemic heart disease and heart failure, causing acute heart failure, pulmonary edema, or destabilization of chronic ischemic heart disease, leading to myocardial infarction or sudden cardiac death [13] [15]. Influenza vaccination reduces the risk

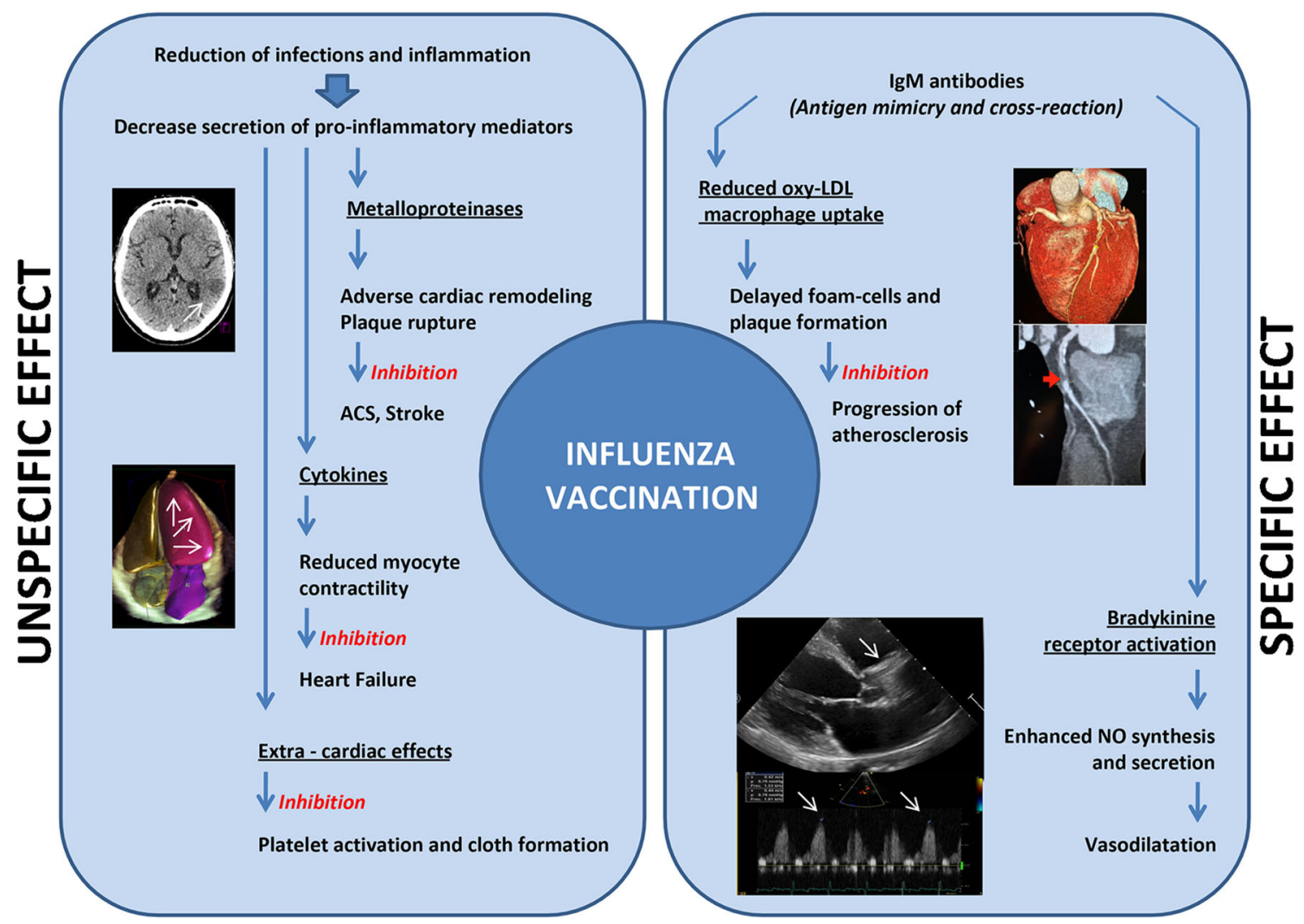

Fig. 1 Possible cardioprotective mechanisms of influenza vaccination 
of infection and inflammation by decreasing the secretion of pro-inflammatory mediators, such as cytokines (that cause reduced myocardial contractility) and metalloproteinases (that cause adverse cardiac remodeling and plaque rupture); influenza vaccination also causes inhibition of platelet activation and cloth formation [13] [43].

The specific mechanism takes into account the immunological properties of the vaccine. The protective effect of the influenza vaccine has been demonstrated in multiple studies. To explain the pleiotropic effect of the influenza vaccine, the "antigen mimicry" between atherothrombotic plaque and influenza virus has been proposed [22]. It has also been proposed that there is an autoimmune "cross-reaction" between influenza and atherosclerosis [13] [44].

Figure 1 summarizes the possible cardioprotective mechanism of influenza vaccination [13].

\section{Conclusion}

It is well studied that obese patients can develop cardiovascular diseases; however, it is less known that the lowinflammatory chronic state might affect host defense and immune cell dysfunction and infections, such as influenza A, could have detrimental outcomes in such patients, such as an increased risk, length of hospitalization, disease severity, morbidity, and mortality.

Cardiovascular diseases, such as ischemic heart disease and heart failure combined with influenza A infection, can trigger acute heart failure exacerbations that increase the overall mortality in a hospitalized setting. Cardiovascular complications associated with influenza infection include myocarditis, pericardial effusion, myopericarditis, right and left ventricle dysfunction, myocardial infarction, heart failure, stroke, and circulatory failure due to septic shock. There have been described several mechanisms by which influenza increases the risk of cardiovascular events; they might be related to pro-inflammatory mediators, sympathetic stimulation, and activation of the coagulation cascade.

While influenza vaccination is associated with a significant reduction in all-cause mortality risk in patients with heart failure, this cardioprotective mechanism may not function as intended in the obese population since they do not always respond optimally to vaccination. Therefore, in an effort to prevent these complications and in the absence of special consideration treatments for this population, we strongly suggest a weight-loss approach. Future studies should focus on developing targeted treatments that can combat the reduced immunocompetence that excess adiposity causes to the patient.

\section{References}

Papers of particular interest, published recently, have been highlighted as:

- Of importance

1. Honce R, Schultz-Cherry S. Impact of obesity on influenza A virus pathogenesis, immune response, and evolution. Front Immunol. 2019;10(MAY). https://doi.org/10.3389/fimmu.2019.01071. This article reviews how an excess of adipose tissue has effects on immunity, it cpomplements greatly our review with a more cellular approach.

2. Milner JJ, Beck MA. The impact of obesity on the immune response to infection. In: Proceedings of the Nutrition Societ. Proc Nutr Soc; 2012;71(2):298-306. https://doi.org/10.1017/ S0029665112000158

3. Obesity and overweight [Internet]. [cited 2019 Oct 21]. Available from: https://www.who.int/news-room/fact-sheets/detail/obesityand-overweight. Accessed Oct 212019.

4. Rojas-Osornio SA, Cruz-Hernández TR, Drago-Serrano ME, Campos-Rodríguez R. Immunity to influenza: impact of obesity. Obes Res Clin Pract [Internet]. 2019;13(5):419-429 [cited 2019 Oct 17]; Available from: http://www.ncbi.nlm.nih.gov/pubmed/ 31542241. Accessed: Oct 17 2019; https://doi.org/10.1016/j.orcp. 2019.05.003

5. Martín V, Castilla J, Godoy P, Delgado-Rodríguez M, Soldevila N, Fernández-Villa T, et al. High body mass index as a risk factor for hospitalization due to influenza: a case-control study. Arch Bronconeumol [Internet]. 2016;52(6):299-307 [cited 2019 Oct 22]; Available from: http://www.ncbi.nlm.nih.gov/pubmed/ 26809749. Accessed Oct 222019.

6. Martin ET, Archer C, McRoberts J, Kulik J, Thurston T, Lephart P, et al. Epidemiology of severe influenza outcomes among adult patients with obesity in Detroit, Michigan, 2011. Influenza Other Respi Viruses [Internet]. 2013;7(6):1004-7 [cited 2019 Oct 22]; Available from: http://www.ncbi.nlm.nih.gov/pubmed/23594280. Accessed Oct 222019.

7. Thomas-Valdés S, Tostes M das GV, Anunciação PC, da Silva BP, HMP S'A. Association between vitamin deficiency and metabolic disorders related to obesity. Crit Rev Food Sci Nutr [Internet]. 2017;57(15):3332-43 [cited 2020 Mar 18]; Available from: http:// www.ncbi.nlm.nih.gov/pubmed/26745150. Accessed Mar 182019

8. Domingo E. RNA virus evolution, population dynamics, and nutritional status. In: Biological trace element research: Humana Press Inc.; Biol Trace Elem Res, 1997;56(1):23-30. https://doi.org/10. 1007/BF02778981

9. Maier HE, Lopez R, Sanchez N, Ng S, Gresh L, Ojeda S, et al. Obesity increases the duration of influenza A virus shedding in adults. J Infect Dis [Internet]. 2018;218(9):1378-82 [cited 2020 Mar 19]; Available from: http://www.ncbi.nlm.nih.gov/pubmed/ 30085119. Accessed Mar 182019

10. Karlsson EA, Hertz T, Johnson C, Mehle A, Krammer F, SchultzCherry S. Obesity outweighs protection conferred by adjuvanted influenza vaccination. MBio [Internet]. 2016;7(4):e01144-16 [cited 2020 Mar 19]; Available from: http://www.ncbi.nlm.nih.gov/ pubmed/27486196. Accessed Mar 19 2019; https://doi.org/10. 1002/14651858.CD005050.pub3

11. Sheridan PA, Paich HA, Handy J, Karlsson EA, Hudgens MG, Sammon AB, et al. Obesity is associated with impaired immune response to influenza vaccination in humans. Int J Obes [Internet]. 2012;36(8):1072-7. [cited 2020 Mar 19]; Available from:. https:// doi.org/10.1038/ijo.2011.208.

12. Neidich SD, Green WD, Rebeles J, Karlsson EA, Schultz-Cherry S, Noah TL, et al. Increased risk of influenza among vaccinated adults 
who are obese. Int J Obes [Internet]. 2017;41(9):1324-30 [cited 2020 Mar 19]; Available from: http://www.ncbi.nlm.nih.gov/ pubmed/28584297. Accessed Mar 192019.

13. Ciszewski A. Cardioprotective effect of influenza and pneumococcal vaccination in patients with cardiovascular diseases [Internet]. Vaccine. 2018;36:202-6 [cited 2019 Oct 15]. Available from: https://linkinghub.elsevier.com/retrieve/pii/S0264410X17317218. Accessed Oct 152019.

14. Clar C, Oseni Z, Flowers N, Keshtkar-Jahromi M, Rees K. Influenza vaccines for preventing cardiovascular disease. Cochrane Database Syst Rev [Internet]. 2015;(5):CD005050 [cited 2019 Oct 15] Available from: http://www.ncbi.nlm.nih.gov/ pubmed/25940444. Accessed Mar 192019.

15. Udell JA, Zawi R, Bhatt DL, Keshtkar-Jahromi M, Gaughran F, Phrommintikul A, et al. Association between influenza vaccination and cardiovascular outcomes in high-risk patients. JAMA [Internet]. 2013;310(16):1711 [cited 2019 Oct 15]; Available from: http:// www.ncbi.nlm.nih.gov/pubmed/24150467. Accessed Oct 152019.

16. Musher DM, Abers MS, Corrales-Medina VF. Acute infection and myocardial infarction. N Engl J Med. 2019;380(2):171-6.

17. Kwong JC, Schwartz KL, Campitelli MA, Chung H, Crowcroft NS, Karnauchow T, et al. Acute myocardial infarction after laboratoryconfirmed influenza infection. N Engl J Med. 2018;378(4):345-53.

18. Fagnoul D, Pasquier P, Bodson L, Ortiz JA, Vincent JL, De Backer D. Myocardial dysfunction during H1N1 influenza infection. J Crit Care. 2013;28(4):321-7.

19. Fountoulaki K, Tsiodras S, Polyzogopoulou E, Olympios C, Parissis J. Beneficial effects of vaccination on cardiovascular events: myocardial infarction, stroke, heart failure. Cardiol. 2018;141(2):98-106.

20. Mamas MA, Fraser D, Neyses L. Cardiovascular manifestations associated with influenza virus infection. Int J Cardiol. 2008;130:304-9.

21. Bergh C, Fall K, Udumyan R, Sjöqvist H, Fröbert O, Montgomery $\mathrm{S}$. Severe infections and subsequent delayed cardiovascular disease. Eur J Prev Cardiol. 2017;24(18):1958-66.

22. Madjid M, Naghavi M, Litovsky S, Casscells SW. Influenza and cardiovascular disease: a new opportunity for prevention and the need for further studies. Circulation [Internet]. 2003;108(22):2730 6 [cited 2019 Dec 27]; Available from: http://www.ncbi.nlm.nih. gov/pubmed/14610013. Accessed Dec 272019.

23. Thygesen K, Alpert JS, Jaffe AS, Chaitman BR, Bax JJ, Morrow DA, et al. Fourth universal definition of myocardial infarction (2018). J Am Coll Cardiol. 2018;72(18):2231-64.

24. Mauriello A, Sangiorgi G, Fratoni S, Palmieri G, Bonanno E, Anemona L, et al. Diffuse and active inflammation occurs in both vulnerable and stable plaques of the entire coronary tree: a histopathologic study of patients dying of acute myocardial infarction. J Am Coll Cardiol. 2005;45(10):1585-93.

25. Rose JJ, Voora D, Cyr DD, Lucas JE, Zaas AK, Woods CW, et al. Gene expression profiles link respiratory viral infection, platelet response to aspirin, and acute myocardial infarction. Schulz C, editor. PLoS One [Internet]. 2015 10(7):e0132259. [cited 2020 Jan 12];Available from: https://dx.plos.org/10.1371/journal.pone. 0132259. Accessed Jan 122019.

26. Semeraro N, Ammollo CT, Semeraro F, Colucci M. Sepsis, thrombosis and organ dysfunction. Thromb Res. 2012;129:290-5.

27. Panhwar MS, Kalra A, Gupta T, Kolte D, Khera S, Bhatt D, et al. Relation of concomitant heart failure to outcomes in patients hospitalized with influenza. Am J Cardiol. 2019;123(9):1478-80.

28. Kytömaa S, Hegde S, Claggett B, Udell JA, Rosamond W, Temte J, et al. Association of influenza-like illness activity with hospitalizations for heart failure: the atherosclerosis risk in communities study. JAMA Cardiol. 2019;4(4):363-9.

29. Vardeny O, Sweitzer NK, Detry MA, Moran JM, Johnson MR, Hayney MS. Decreased immune responses to influenza vaccination in patients with heart failure. J Card Fail. 2009;15(4):368-73.
30. Kindermann I, Barth C, Mahfoud F, Ukena C, Lenski M, Yilmaz A, et al. Update on myocarditis. J Am Coll Cardiol Elsevier USA. 2012;59:779-92. https://doi.org/10.1016/j.jacc.2011.09.074

31. Caforio ALP, Pankuweit S, Arbustini E, Basso C, Gimeno-Blanes J, Felix SB, et al. Current state of knowledge on aetiology, diagnosis, management, and therapy of myocarditis: a position statement of the European Society of Cardiology Working Group on Myocardial and Pericardial Diseases. Eur Heart J. 2013;34(33): 2636-48.

32. Tschöpe C, Cooper LT, Torre-Amione G, Van Linthout S. Management of myocarditis-related cardiomyopathy in adults. Circ Res. 2019;124(11):1568-83.

33. Nichol KL, Nordin J, Mullooly J, Lask R, Fillbrandt K, Iwane M. Influenza vaccination and reduction in hospitalizations for cardiac disease and stroke among the elderly. N Engl J Med. 2003;348(14): 1322-32.

34. Toschke AM, Heuschmann PU, Wood O, Wolfe CDA. Temporal relationship between influenza infections and subsequent first-ever stroke incidence. Age Ageing. 2009;38:100-3.

35. Boehme AK, Luna J, Kulick ER, Kamel H, Elkind MSV. Influenza-like illness as a trigger for ischemic stroke. Ann Clin Transl Neurol. 2018;5(4):456-63.

36. Urbanek C, Palm F, Grau A. Influenza and stroke risk: a key target not to be missed? Infect Disord-Drug Targets [Internet]. 2010;10(2):122-31 [cited 2020 Jan 12]; Available from: http:// www.eurekaselect.com/openurl/content.php?genre=article\&issn= $1871-5265 \&$ volume $=10 \&$ issue $=2 \&$ spage $=122$. Accessed Jan 12 2019.

37. Muhammad S, Haasbach E, Kotchourko M, Strigli A, Krenz A, Ridder DA, et al. Influenza virus infection aggravates stroke outcome. Stroke. 2011;42(3):783-91.

38. Piepoli MF, Hoes AW, Agewall S, Albus C, Brotons C, Catapano $\mathrm{AL}$, et al. 2016 European guidelines on cardiovascular disease prevention in clinical practice. The Sixth Joint Task Force of the European Society of Cardiology and Other Societies on Cardiovascular Disease Prevention in Clinical Practice (constituted by representatives of 10 societies and by invited experts. Developed with the special contribution of the European Association for Cardiovascular Prevention \& RehabilitationG Ital Cardiol (Rome). 2017;18(7):547-612.

39. Smeeth L, Thomas SL, Hall AJ, Hubbard R, Farrington P, Vallance P. Risk of myocardial infarction and stroke after acute infection or vaccination. N Engl J Med. 2004;351(25):2611-8.

40. Corrales-Medina VF, Alvarez KN, Weissfeld LA, Angus DC, Chirinos JA, Chang CCH, et al. Association between hospitalization for pneumonia and subsequent risk of cardiovascular disease. JAMA. 2015;313(3):264-74.

41. Yende S, D'Angelo G, Kellum JA, Weissfeld L, Fine J, Welch RD, et al. Inflammatory markers at hospital discharge predict subsequent mortality after pneumonia and sepsis. Am J Respir Crit Care Med. 2008;177(11):1242-7.

42. Vardeny O, Claggett B, Udell JA, Packer M, Zile M, Rouleau J, et al. Influenza vaccination in patients with chronic heart failure: the PARADIGM-HF trial. JACC Hear Fail. 2016;4(2):152-8.

43. Naghavi M, Libby P, Falk E, Casscells SW, Litovsky S, Rumberger $\mathrm{J}$, et al. From vulnerable plaque to vulnerable patient: a call for new definitions and risk assessment strategies: part II. Circulation. 2003;108:1772-8.

44. Veljkovic V, Glisic S, Veljkovic N, Bojic T, Dietrich U, Perovic $\mathrm{VR}$, et al. Influenza vaccine as prevention for cardiovascular diseases: possible molecular mechanism. Vaccine. 2014;32(48):656975 .

Publisher's Note Springer Nature remains neutral with regard to jurisdictional claims in published maps and institutional affiliations. 